\title{
Excitonic linewidth and coherence lifetime in monolayer transition metal dichalcogenides
}

Malte Selig

Gunnar Berghäuser

Archana Raja

Philipp Nagler

Christian Schüller

Tony F. Heinz

Tobias Korn

Alexey Chernikov

Ermin Malic

Andreas Knorr 


\title{
Excitonic linewidth and coherence lifetime in monolayer transition metal dichalcogenides
}

\author{
Malte Selig ${ }^{1}$, Gunnar Berghäuser ${ }^{1}$, Archana Raja ${ }^{2,3}$, Philipp Nagler ${ }^{4}$, Christian Schüller ${ }^{4}$, \\ Tony F. Heinz ${ }^{3,5,6}$, Tobias Korn ${ }^{4}$, Alexey Chernikov ${ }^{4,6}$, Ermin Malic ${ }^{7}$, and Andreas \\ Knorr $^{1}$ \\ ${ }^{1}$ Institut für Theoretische Physik, Technische Universität Berlin, 10623 Berlin, Germany \\ ${ }^{2}$ Department of Chemistry, Columbia University, New York, New York 10027, USA \\ ${ }^{3}$ Department of Applied Physics, Stanford University, Stanford, California 94305, USA \\ ${ }^{4}$ Institut für Experimentelle und Angewandte Physik, Universität Regensburg, 93040 \\ Regensburg, Germany \\ ${ }^{5}$ SLAC National Accelerator Laboratory, Menlo Park, California 94025, USA \\ ${ }^{6}$ Departments of Physics and Electrical Engineering, Columbia University, New York, \\ New York 10027, USA \\ ${ }^{7}$ Chalmers University of Technology, Department of Physics, SE-412 96 Gothenburg, \\ Sweden
}

\begin{abstract}
Monolayers of transition metal dichalcogenides are direct gap semiconductors, which have attracted much attention in the recent past. Due to a strong Coulomb interaction, they possess strongly bound electron-hole pairs, with binding energies of hundreds of $\mathrm{meV}$ which is an order of magnitude larger than in conventional materials. Here, we investigate the microscopic origin of the homogeneous linewidth and coherence lifetime of excitonic resonances in monolayer molybdenum disulfide, taking exciton phonon scattering and radiative recombination into account. We find a superlinear increasing homogeneous linewidth from $2 \mathrm{meV}$ at $5 \mathrm{~K}$ to $14 \mathrm{meV}$ at room temperature corresponding to a coherence lifetime of $160 \mathrm{fs}$ and $25 \mathrm{fs}$.
\end{abstract}

\section{Introduction}

Atomically thin transition metal dichalcogenides (TMDs) have attracted much attention in current research. While bulk TMDs are indirect semiconductors, monolayer TMDs possess direct band gaps at the $K$ and $K^{\prime}$ valleys in the Brillouin zone. ${ }^{1-4}$ In contrast to conventional semiconductors, TMDs are characterized by an extraordinarily strong Coulomb interaction giving rise to the formation of excitons and even trions with binding energies in the range of several hundreds of $\mathrm{meV}^{5-8}$ As a result, their optical spectra are dominated by excitonic effects. ${ }^{9,10}$ The homogeneous linewidth of excitonic resonances has been investigated experimentally ${ }^{11,12}$ and theoretically, ${ }^{13}$ pointing out that in tungsten based TMDs, dark exciton states where electron and hole are located at different high symmetry point in the first Brillouin zone are located energetically below the optical bright ones. Thus they contribute to the homogeneous linewidth as an efficient scattering channel.

In this work, we present a microscopic study on the elementary processes behind the observed homogeneous linewidth in monolayer molybdenum disulfide. In particular, we investigate the influence of radiative recombination ${ }^{11,13,14}$ and exciton-phonon scattering ${ }^{13,15}$ including their dependence on temperature.

Ultrafast Phenomena and Nanophotonics XXI, edited by Markus Betz, Abdulhakem Y. Elezzabi, Proc. of SPIE Vol. 10102, 101021F · () 2017 SPIE · CCC code: 0277-786X/17/\$18 doi: $10.1117 / 12.2252486$ 


\section{Theoretical Model}

The starting point of our study is the calculation of the absorption coefficient ${ }^{16}$

$$
\alpha(\omega)=\frac{\omega}{n c_{0}} \Im(\chi(\omega))=\frac{\omega}{n c_{0}} \Im\left(\frac{j^{\sigma_{-}}(\omega)}{\epsilon_{0} \omega^{2} A^{\sigma-}(\omega)}\right),
$$

with the light frequency $\omega$, the refractive index $n$, the vacuum speed of light $c_{0}$, the vector potential of the incident light $A^{\sigma_{-}}(\omega)$, the vacuum permittivity $\epsilon_{0}$, and finally the optical susceptibility $\chi(\omega)=\frac{j^{\sigma}-(\omega)}{\epsilon_{0} \omega^{2} A^{\sigma}-(\omega)}$. The latter is determined by the current density $j^{\sigma_{-}}(\omega)=\mathbf{j}(\omega) \cdot(1,-i)$. Here, we restrict the study to one excitonic transitions at the $K$-point, which are excited by right-handed circular polarized incoming light. The optically induced current reads

$$
j^{\sigma_{-}}(\omega)=\sum_{\mathbf{q}}\left(M_{\mathbf{q}}^{\sigma_{-} *} \varphi_{\mathbf{q}}^{\mu} P_{\mathbf{0}}^{\mu}(\omega)+\text { c.c. }\right),
$$

with the optical matrix element $M_{\mathbf{q}}^{\sigma_{-}}$and the Fourier transform of the microscopic polarization $P_{\mathbf{0}}(\omega)$. The latter stands for the more general excitonic polarization $P_{\mathbf{Q}}(\omega)$, where $\mathbf{Q}$ is the Fourier component of the electron-hole center-of-mass motion in real space. The appearing excitonic wavefunction $\varphi_{\mathbf{q}}^{\mu}$ depends on the momentum $\mathbf{q}$ for the relative motion of the electrons and holes in real space and the exciton state $\mu$ and can be obtained by solving the Wannier equation ${ }^{17-19}$

$$
\frac{\hbar^{2} \mathbf{q}^{2}}{2 m_{0}} \varphi_{\mathbf{q}}^{\mu}-\sum_{\mathbf{k}} V_{\mathbf{q}, \mathbf{k}}^{e x c} \varphi_{\mathbf{q}+\mathbf{k}}^{\mu}+E_{g a p} \varphi_{\mathbf{q}}^{\mu}=E^{\mu} \varphi_{\mathbf{q}}^{\mu},
$$

with the excitonic energy $E^{\mu}$ and the excitonic part of the Coulomb interaction $V_{\mathbf{q}, \mathbf{k}}^{\text {exc }}$.

The absorption coefficient from Eq. (1) is determined by the microscopic polarization in the excitonic basis which will be discussed in the following. Our quantity of interest is the microscopic polarization in the electron picture $P_{\mathbf{k}_{\mathbf{1}}, \mathbf{k}_{\mathbf{2}}}=\left\langle a_{\mathbf{k}_{\mathbf{1}}}^{\dagger v} a_{\mathbf{k}_{\mathbf{2}}}^{c}\right\rangle$ with electron annihilation (creation) operators $a_{\mathbf{k}}^{(\dagger) \lambda}$ with momentum $k$ and band index $\lambda=c, v$, from which we will obtain an expression for the excitonic polarization later. Applying the Heisenbergs equation of motion $i \hbar \partial_{t} \mathcal{P}_{\mathbf{k}_{\mathbf{1}}, \mathbf{k}_{\mathbf{2}}}=\left[H, \mathcal{P}_{\mathbf{k}_{\mathbf{1}}, \mathbf{k}_{\mathbf{2}}}\right]$, we can determine the temporal evolution of the microscopic polarization $P_{\mathbf{k}_{1}, \mathbf{k}_{2}}^{\mu}$ which will be transformed into the excitonic picture later. The appearing Hamilton operator $H=H_{0}+H_{c-l}+H_{c-c}+H_{c-p h}$ which we take from ${ }^{13}$ includes (i) the interaction-free contribution $H_{0}$ that contains the dispersion of electrons and phonons, (ii) the carrier-light interaction $H_{e l-l}$, (iii) the carrier-carrier interaction $H_{c-c}$, and (iv) the carrier-phonon interaction $H_{c-p h}$.

The first step is to compute the equation of motion for the microscopic polarization $P_{\mathbf{k}_{\mathbf{1}}, \mathbf{k}_{\mathbf{2}}}=\left\langle a_{\mathbf{k}_{\mathbf{1}}}^{\dagger v} a_{\mathbf{k}_{\mathbf{2}}}^{c}\right\rangle$, which results in the so called semiconductor Bloch equation ${ }^{17,20}$

$$
\begin{aligned}
i \hbar \partial_{t} P_{\mathbf{k}_{\mathbf{1}}, \mathbf{k}_{\mathbf{2}}} & =\left(\epsilon_{c}\left(\mathbf{k}_{2}\right)-\epsilon_{v}\left(\mathbf{k}_{1}\right)\right) P_{\mathbf{k}_{\mathbf{1}} \mathbf{k}_{\mathbf{2}}}+\frac{i \hbar e}{m} \mathbf{M}_{\mathbf{k}_{\mathbf{2}}}^{c v} \cdot \mathbf{A}\left(1-f_{\mathbf{k}_{\mathbf{1}}}^{h}-f_{\mathbf{k}_{\mathbf{2}}}^{e}\right) \delta_{\mathbf{k}_{\mathbf{1}}, \mathbf{k}_{\mathbf{2}}} \\
& +\sum_{\mathbf{k}} V_{\mathbf{k}_{\mathbf{1}}, \mathbf{k}_{\mathbf{2}}, \mathbf{k}}^{r e n o m}\left(f^{e}, f^{h}\right) P_{\mathbf{k}_{\mathbf{1}}, \mathbf{k}_{\mathbf{2}}}-\left(1-f_{\mathbf{k}_{\mathbf{2}}}^{e}-f_{\mathbf{k}_{\mathbf{1}}}^{h}\right) \sum_{\mathbf{k}} V_{\mathbf{k}_{\mathbf{1}}, \mathbf{k}_{\mathbf{2}}, \mathbf{k}}^{e x c} P_{\mathbf{k}_{\mathbf{1}}+\mathbf{k}, \mathbf{k}_{\mathbf{2}}+\mathbf{k}} \\
& +\sum_{\mathbf{q}, \alpha} g_{\mathbf{k}_{\mathbf{2}}, \mathbf{k}_{\mathbf{2}}-\mathbf{q}, \mathbf{q}}^{c \alpha}\left(S_{\mathbf{k}_{\mathbf{1}}, \mathbf{k}_{\mathbf{2}}-\mathbf{q}, \mathbf{q}}^{\alpha}+\tilde{S}_{\mathbf{k}_{\mathbf{1}}, \mathbf{k}_{\mathbf{2}}-\mathbf{q},-\mathbf{q}}^{\alpha}\right)-g_{\mathbf{k}_{\mathbf{1}}+\mathbf{q}, \mathbf{k}_{1}, \mathbf{q}}^{v \alpha}\left(S_{\mathbf{k}_{\mathbf{1}}+\mathbf{q}, \mathbf{k}_{\mathbf{2}}, \mathbf{q}}^{\alpha}+\tilde{S}_{\mathbf{k}_{\mathbf{1}}+\mathbf{q}, \mathbf{k}_{\mathbf{2}},-\mathbf{q}}^{\alpha}\right) .
\end{aligned}
$$


Here, we treated the upcoming hierarchy problem (2-point quantities couple to 4-point quantities via Coulomb interaction) within the Hartree Fock approximation. ${ }^{17}$ The first line is known as the optical Bloch equation, where the first term describes the oscillation of the microscopic polarization with the band gap energy and the second term contributes to the optical excitation of a the microscopic polarization with an external electric field. The Kronecker delta accounts for the vanishing photon momentum, meaning that only polarizations with same electron and hole momentum can be excited optically. The phase space filling factor blocks the optical excitation when electron and hole densities are excited. The second line contributes to the Coulomb interaction, where the first term renormalizes the band gap energy. The second term leads to the formation of bound electron hole pairs. The thirds line, stemming from the electron phonon interaction couples the microscopic polarization to so called phonon assisted polarization $S_{\mathbf{k}_{1}, \mathbf{k}_{2}, \mathbf{q}}^{\alpha}=\left\langle a_{\mathbf{k}_{1}}^{\dagger v} a_{\mathbf{k}_{\mathbf{2}}}^{c} b_{\mathbf{q}}^{\alpha}\right\rangle$ and $\tilde{S}_{\mathbf{k}_{1}, \mathbf{k}_{2}, \mathbf{q}}^{\alpha}=\left\langle a_{\mathbf{k}_{1}}^{\dagger v} a_{\mathbf{k}_{2}}^{c} b_{\mathbf{q}}^{\dagger \alpha}\right\rangle$. For these quantities, we derive again a equation of motion

$$
\begin{aligned}
& i \hbar \partial_{t} S_{\mathbf{k}_{1}+\mathbf{q}, \mathbf{k}_{\mathbf{2}}, \mathbf{q}}^{v c \alpha}=\left(\epsilon_{\mathbf{k}_{\mathbf{2}}}^{c}-\epsilon_{\mathbf{k}_{\mathbf{1}}+\mathbf{q}}^{v}+E_{\mathbf{q}}^{\alpha}+\sum_{\mathbf{k}} V_{\mathbf{k}_{\mathbf{1}}+\mathbf{q}, \mathbf{k}_{2}, \mathbf{k}}^{r e n o m}\right) S_{\mathbf{k}_{1}+\mathbf{q}, \mathbf{k}_{\mathbf{2}}, \mathbf{q}}^{v c \alpha}+ \\
& +\sum_{\mathbf{k}} V_{\mathbf{k}_{1}+\mathbf{q}, \mathbf{k}_{2}, \mathbf{k}}^{e x c}\left(1-f_{\mathbf{k}_{2}}^{e}-f_{\mathbf{k}_{1}+\mathbf{q}}^{h}\right) S_{\mathbf{k}_{1}+\mathbf{k}+\mathbf{q}, \mathbf{k}_{\mathbf{2}}+\mathbf{k}, \mathbf{q}}^{v c \alpha}+ \\
& +\left(g_{\mathbf{k}_{2}, \mathbf{k}_{2}+\mathbf{q},-\mathbf{q}}^{c \alpha}\left(1-f_{\mathbf{k}_{\mathbf{2}}}^{e}\right) P_{\mathbf{k}_{1}+\mathbf{q}, \mathbf{k}_{\mathbf{2}}+\mathbf{q}}^{v c}\right. \\
& \left.-g_{\mathbf{k}_{1}, \mathbf{k}_{1}+\mathbf{q},-\mathbf{q}}^{v \alpha}\left(1-f_{\mathbf{k}_{\mathbf{1}}+\mathbf{q}}^{h}\right) P_{\mathbf{k}_{\mathbf{1}}, \mathbf{k}_{\mathbf{2}}}^{v c}\right)\left(1+n_{\mathbf{q}}^{\alpha}\right)+ \\
& +\left(g_{\mathbf{k}_{\mathbf{2}}, \mathbf{k}_{\mathbf{2}}+\mathbf{q},-\mathbf{q}}^{c \alpha} f_{\mathbf{k}_{\mathbf{2}}}^{e} P_{\mathbf{k}_{\mathbf{1}}+\mathbf{q}, \mathbf{k}_{\mathbf{2}}+\mathbf{q}}^{v c}-g_{\mathbf{k}_{1}, \mathbf{k}_{1}+\mathbf{q},-\mathbf{q}}^{v \alpha} f_{\mathbf{k}_{1}+\mathbf{q}}^{h} P_{\mathbf{k}_{1}, \mathbf{k}_{\mathbf{2}}}^{v c}\right) n_{\mathbf{q}}^{\alpha}+ \\
& i \hbar \partial_{t} \tilde{S}_{\mathbf{k}_{\mathbf{1}}+\mathbf{q}, \mathbf{k}_{\mathbf{2}},-\mathbf{q}}^{v \alpha}=\left(\epsilon_{\mathbf{k}_{\mathbf{2}}}^{c}-\epsilon_{\mathbf{k}_{\mathbf{1}}+\mathbf{q}}^{v}-E_{-\mathbf{q}}^{\alpha}+\sum_{\mathbf{k}} V_{\mathbf{k}_{\mathbf{1}}+\mathbf{q}, \mathbf{k}_{\mathbf{2}}, \mathbf{k}}^{r e o m}\right) \tilde{S}_{\mathbf{k}_{\mathbf{1}}+\mathbf{q}, \mathbf{k}_{\mathbf{2}},-\mathbf{q}}^{v c \alpha}+ \\
& +\sum_{\mathbf{k}} V_{\mathbf{k}_{1}+\mathbf{q}, \mathbf{k}_{2}, \mathbf{k}}^{e x c}\left(1-f_{\mathbf{k}_{\mathbf{2}}}^{e}-f_{\mathbf{k}_{1}+\mathbf{q}}^{h}\right) \tilde{S}_{\mathbf{k}_{1}+\mathbf{k}+\mathbf{q}, \mathbf{k}_{\mathbf{2}}+\mathbf{k},-\mathbf{q}}+ \\
& +\left(g_{\mathbf{k}_{\mathbf{2}}, \mathbf{k}_{\mathbf{2}}+\mathbf{q},-\mathbf{q}}^{c \alpha}\left(1-f_{\mathbf{k}_{\mathbf{2}}}^{e}\right) P_{\mathbf{k}_{\mathbf{1}}+\mathbf{q}, \mathbf{k}_{\mathbf{2}}+\mathbf{q}}^{v c}\right. \\
& \left.-g_{\mathbf{k}_{\mathbf{1}}, \mathbf{k}_{\mathbf{1}}+\mathbf{q},-\mathbf{q}}^{v \alpha}\left(1-f_{\mathbf{k}_{\mathbf{1}}+\mathbf{q}}^{h}\right) P_{\mathbf{k}_{\mathbf{1}}, \mathbf{k}_{\mathbf{2}}}^{v c}\right) n_{\mathbf{q}}^{\alpha}+ \\
& +\left(g_{\mathbf{k}_{\mathbf{2}}, \mathbf{k}_{\mathbf{2}}+\mathbf{q},-\mathbf{q}}^{c \alpha} f_{\mathbf{k}_{\mathbf{2}}}^{e} P_{\mathbf{k}_{\mathbf{1}}+\mathbf{q}, \mathbf{k}_{\mathbf{2}}+\mathbf{q}}^{v c}-g_{\mathbf{k}_{\mathbf{1}}, \mathbf{k}_{\mathbf{1}}+\mathbf{q},-\mathbf{q}}^{v \alpha} f_{\mathbf{k}_{\mathbf{1}}+\mathbf{q}}^{h} P_{\mathbf{k}_{1}, \mathbf{k}_{\mathbf{2}}}^{v c}\right)\left(1+n_{\mathbf{q}}^{\alpha}\right) .
\end{aligned}
$$

Here in both equations, the first line describes the oscillation of the phonon assisted quantities with the renormalized band gap energy and the phonon energy $E_{\mathbf{q}}^{\alpha}$. The second line accounts for the formation of bound electron hole pairs. The last three lines in both equations stem from the electron phonon interaction. Here terms proportional to the phonon occupation $n_{\mathbf{q}}^{\alpha}$ account for phonon absorption processes, whereas terms proportional to $1+n_{\mathbf{q}}^{\alpha}$ account for phonon emission processes. Further terms proportional to electron/ hole occupation $f^{e / h}$ describe in-scattering processes, whereas terms proportional to $1-f^{e / h}$ describe outscattering. The blocking factor accounts for the Pauli principle, which means that scattering only occurs, if the final state is empty. In the following we will a assume a undoped material under low excitations, where we can neglect density dependent effects.

The next step is to transform the equations of motion for the microscopic polarizations and the phonon assisted polarizations to the excitonic picture. Therefore we first introduce center of mass coordinates

$$
\begin{aligned}
& \mathbf{k}_{1}=\mathbf{q}+\frac{m_{h}}{m_{h}+m_{e}} \mathbf{Q}=\mathbf{q}+\beta \mathbf{Q} \\
& \mathbf{k}_{2}=\mathbf{q}-m_{e}-\begin{array}{c}
m_{h}+m_{e} \\
m_{h}
\end{array}=\mathbf{q}-\alpha \mathbf{Q} .
\end{aligned}
$$

with the Fourier component of the relative motion $q$ and the Fourier component of the center of mass motion $\mathbf{Q}$. The next step is to write the microscopic polarization in center of mass coordinates $P_{\mathbf{q}, \mathbf{Q}}$ as a decomposition of excitonic eigenfunctions $P_{\mathbf{q}, \mathbf{Q}}=\sum_{\lambda} \varphi_{\mathbf{q}, \mathbf{Q}}^{\lambda} P_{\mathbf{Q}}^{\lambda}$, where we assume that the exciton wavefunction does not depend on the center of mass momentum $\varphi_{\mathbf{q}, \mathbf{Q}}^{\lambda}=\varphi_{\mathbf{q}}^{\lambda}$. For the equations 4, 5 and 6 we obtain 


$$
\begin{aligned}
i \hbar \partial_{t} P_{\mathbf{Q}}^{\mu} & =\left(\frac{\hbar^{2} \mathbf{Q}^{2}}{2 M}+E^{\mu}\right) P_{\mathbf{Q}}^{\mu}+\frac{i \hbar e}{m} \sum_{\mathbf{q}} \varphi_{\mathbf{q}}^{* \mu} \mathbf{M}_{\mathbf{q}}^{c v} \cdot \mathbf{A} \delta_{\mathbf{Q}, \mathbf{0}} \\
& +\sum_{\mathbf{q}^{\prime}, \alpha, \lambda} g_{\mathbf{q}^{\prime}}^{\mu \lambda \alpha}\left(S_{\mathbf{Q}^{\prime}+\mathbf{q}^{\prime}, \mathbf{q}^{\prime}}^{\lambda \alpha}+\tilde{S}_{\mathbf{Q}^{\prime}+\mathbf{q}^{\prime},-\mathbf{q}^{\prime}}^{\lambda \alpha}\right) \\
i \hbar \partial_{t} S_{\mathbf{Q}+\mathbf{q}^{\prime}, \mathbf{q}^{\prime}}^{\mu \alpha} & =\left(\frac{\hbar^{2}\left(\mathbf{Q}+\mathbf{q}^{\prime}\right)^{2}}{2 M}+E^{\mu}+E_{\mathbf{q}^{\prime}}^{\alpha}\right) S_{\mathbf{Q}+\mathbf{q}^{\prime}, \mathbf{q}^{\prime}}^{\mu \alpha}+\sum_{\lambda, \mathbf{q}^{\prime}} g_{-\mathbf{q}^{\prime}}^{\mu \lambda \alpha}\left(1+n_{\mathbf{q}^{\prime}}^{\alpha}\right) P_{\mathbf{Q}}^{\lambda} \\
i \hbar \partial_{t} \tilde{S}_{\mathbf{Q}+\mathbf{q}^{\prime},-\mathbf{q}^{\prime}}^{\mu \alpha} & =\left(\frac{\hbar^{2}\left(\mathbf{Q}+\mathbf{q}^{\prime}\right)^{2}}{2 M}+E^{\mu}-E_{-\mathbf{q}^{\prime}}^{\alpha}\right) \tilde{S}_{\mathbf{Q}+\mathbf{q}^{\prime},-\mathbf{q}^{\prime}}^{\mu \alpha}+\sum_{\lambda, \mathbf{q}^{\prime}} g_{-\mathbf{q}^{\prime}}^{\mu \lambda \alpha} n_{-\mathbf{q}^{\prime}}^{\alpha} P_{\mathbf{Q}}^{\lambda} .
\end{aligned}
$$

Equation 9 describes the equation of motion of the excitonic polarization for the $\mu^{\prime}$ th exciton state. It oscillates with the kinetic energy $\frac{\hbar^{2} \mathbf{Q}^{2}}{2 M}$ and the exciton energy $E^{\mu}$, which we obtained as a solution of the Wannier equation, equation 3.

The second term in equation 9 describes the excitonic polarization to an incoming electro magnetic field. The third term describes the coupling to the phonon assisted excitonic polarization via exciton phonon coupling. The exciton phonon coupling element reads

$$
g_{\mathbf{q}^{\prime}}^{\mu \lambda \alpha}=\sum_{\mathbf{q}}\left(g_{\mathbf{q}, \mathbf{q}-\mathbf{q}^{\prime}, \mathbf{q}^{\prime}}^{c \alpha} \varphi_{\mathbf{q}}^{L \mu} \varphi_{\mathbf{q}-\beta \mathbf{q}^{\prime}}^{R \lambda}-g_{\mathbf{q}+\mathbf{q}^{\prime}, \mathbf{q}, \mathbf{q}^{\prime}}^{v \alpha} \varphi_{\mathbf{q}}^{L \mu} \varphi_{\mathbf{q}+\alpha \mathbf{q}^{\prime}}^{R \lambda}\right) .
$$

Here, we made the assumption that the exciton phonon coupling does not depend on the center of mass momentum of the excitonic polarization. However, since we are interested in the broadening of the optically accessible excitonic polarization, this treatment is exact.

In the equations 10 and 11 the first term describes the oscillation of the phonon assisted excitonic polarization with the kinetic energy $\frac{\hbar^{2}\left(\mathbf{Q}+\mathbf{q}^{\prime}\right)^{2}}{2 M}$, the excitonic energy $E^{\mu}$ and the phonon energy $E_{\mathbf{q}}^{\alpha}$. The second term, stemming from the exciton phonon coupling, couples the phonon assisted polarizations to the excitonic polarization. To obtain an expression for the dephasing of the excitonic polarization, we treat the system of coupled differential equation 9, 10 and 11 within the Born-Markov approximation. ${ }^{17,21}$

Restricting ourself to the energetically lowest lying 1s exciton, we obtain

$$
\gamma_{\mathbf{Q}}^{1 s}=\frac{\pi}{\hbar^{2}} \sum_{\mathbf{q}, \alpha}\left|g_{\mathbf{q}}^{1 s \alpha}\right|^{2}\left(\left(1+n_{\mathbf{q}}^{\alpha}\right) \delta\left(\frac{\hbar(\mathbf{Q}+\mathbf{q})^{2}}{2 M}-\frac{\hbar \mathbf{Q}^{2}}{2 M}+\omega_{\mathbf{q}}^{\alpha}\right)+n_{-\mathbf{q}}^{\alpha} \delta\left(\frac{\hbar(\mathbf{Q}+\mathbf{q})^{2}}{2 M}-\frac{\hbar \mathbf{Q}^{2}}{2 M}-\omega_{-\mathbf{q}}^{\alpha}\right)\right),
$$

with the phonon frequencies $\omega_{\mathbf{q}}^{\alpha}=\frac{E_{\mathbf{q}}^{\alpha}}{\hbar}$. Here the term proportional to $1+n_{\mathbf{q}}^{\alpha}$ indicates the emission of phonons, whereas term proportional to $n_{\mathbf{q}}^{\alpha}$ indicates the absorption of phonons.

To compute the radiative dephasing rate we have to solve the Maxwell-Bloch-Equations self consistently. We start with the inhomogeneous wave equation

$$
\left(\nabla^{2}-\frac{n^{2}}{c^{2}} \partial_{t}^{2}\right) \mathbf{E}(\mathbf{r}, t)=\mu_{0} \partial_{t} \mathbf{j}(\mathbf{r}, t),
$$

which can be derived from the Maxwell-Equations. Here $\mathbf{E}(\mathbf{r}, t)$ is the electrical field, $\mathbf{j}(\mathbf{r}, t)$ the current density in the material, $n$ is the refractive index of the surrounding medium and $c$ the vacuum speed of light. For the following we assume that the electric field propagates in $z$-direction and is right handed circular polarized. As solution we obtain ${ }^{19}$

$$
E^{\sigma_{-}}(t)=E_{0}^{\sigma_{-}}\left(t-\frac{z}{c}\right)-\frac{c \mu_{0}}{2} j^{\sigma_{-}}\left(t-\frac{\left|z-z_{0}\right|}{c}\right),
$$


with $E_{0}^{\sigma_{-}}\left(t-\frac{z}{c}\right)$ the homogeneous solution and the second term being the inhomogeneous solution of the wave equation describing the effect of the current density in the material to the electric field. Next we insert $E=-\partial_{t} A$ and Fourier transform the equation with $\partial_{t} \rightarrow-i \omega$. Thus we obtain

$$
A(\omega)=A_{0}(\omega)+\frac{i c \mu_{0}}{\omega} j(\omega) .
$$

The macroscopic current density can be expressed in terms of the microscopic polarisation

$$
j^{\sigma_{-}}(\omega)=\sum_{\mathbf{q} \mu}\left(M_{\mathbf{q}}^{\sigma_{-} *} \varphi_{\mathbf{q}}^{R \mu} P^{\mu}(\omega)+\text { c.c. }\right) .
$$

Thus we end up with a expression for the vector potential

$$
A(\omega)=A_{0}(\omega)+\frac{i c \mu_{0}}{\omega} \sum_{\mathbf{q}, \mu}\left(M_{\mathbf{q}}^{\sigma_{-} *} \varphi_{\mathbf{q}}^{R \mu} P^{\mu}(\omega)+c . c .\right) .
$$

This expression of the vector potential includes the incident vector potential $A_{0}(\omega)$ which can be controlled experimentally and the in the $2 \mathrm{~d}-$ material induced vector potential $\frac{i c \mu_{0}}{\omega} \sum_{\mathbf{q}, \mu}\left(M_{\mathbf{q}}^{\sigma_{-}} \varphi_{\mathbf{q}}^{R \mu} P^{\mu}(\omega)+\right.$ c.c. $)$.

To find an expression for $P(\omega)$, we take Eq. 9 and set $\mathbf{Q}=0$, since only transitions with vanishing center of mass momentum contribute to the macroscopic current density. A Fourier transformation leads to

$$
\hbar \omega P^{\mu}(\omega)=E^{\mu} P(\omega)+\frac{i e \hbar}{m} \sum_{\mathbf{q}} M_{\mathbf{q}}^{\sigma_{-}}\left(1-f_{\mathbf{q}}^{e}-f_{\mathbf{q}}^{h}\right) \varphi_{\mathbf{q}}^{L \mu} A(\omega) .
$$

Combining both equations 18,19 and neglecting off-resonant terms with respect to a rotating wave approximation, ${ }^{17}$ we obtain an equation for the microscopic polarisation

$$
P^{\mu}(\omega)=\frac{\frac{i e \hbar}{m} \sum_{\mathbf{q}}\left(1-f_{\mathbf{q}}^{e}-f_{\mathbf{q}}^{h}\right) M_{\mathbf{q}}^{\sigma-} \varphi_{\mathbf{q}}^{L \mu} A_{0}(\omega)}{\hbar \omega-E^{\mu}-i \gamma_{\text {rad }}^{\mu}},
$$

where we identified the radiative dephasing rate

$$
\gamma_{r a d}^{\mu}=\frac{\hbar^{2} e^{2} \mu_{0} c}{\omega m^{2} \frac{n_{1}+n_{2}}{2}}\left|\sum_{\mathbf{q}} M_{\mathbf{q}}^{\sigma-} \varphi_{\mathbf{q}}^{R * \mu}\right|^{2} .
$$

\section{Numerical Results}

Here, we will give numerical results for the homogeneous broadening in monolayer molybdenum disulfide. The bandstructure is treated in effective mass approximation with parameters obtained from. ${ }^{22}$ Longwavelenght acoustic phonons are treated in Debye approximation with parameters obtained from..$^{23,24}$ Optical phonons are treated in Einstein approximation with parameters from. ${ }^{23,24}$ The electron phonon coupling for both phonons modes is treated in an effective deformation potential approximation, which was obtained by fitting an effective deformation potential model to ab initio electron phonon scattering rates. ${ }^{23,24}$ The oscillator strength of the optical matrix element was adjusted to experimental data ${ }^{25}$ following the procedure given in. ${ }^{11}$ In general TMDs exhibit a complex quasi particle bandstructure, ${ }^{22,26}$ resulting in a complex exciton band structure as well, including dark exciton states far beyond the light cone ${ }^{13,27}$ or spin forbidden exciton states. ${ }^{28}$ Further exchange coupling plays a significant role in understanding the complex valley physics in these materials. ${ }^{29,30}$ However in this study, we will focus on the weak excitation limit where only exciton phonon coupling and radiative recombination are contributing to the homogeneous linewidth. In tungsten based materials, exciton states where a hole is placed at the $K$ point and an electron is placed at a $\Lambda$ point in the 1 . BZ are known to be efficient relaxation channels ${ }^{13}$ since they are located energetically below the optical bright state. Here in molybedenum disulfide these state are located approximately $150 \mathrm{meV}$ above the bright states. That is why these states are neglected in the following. 


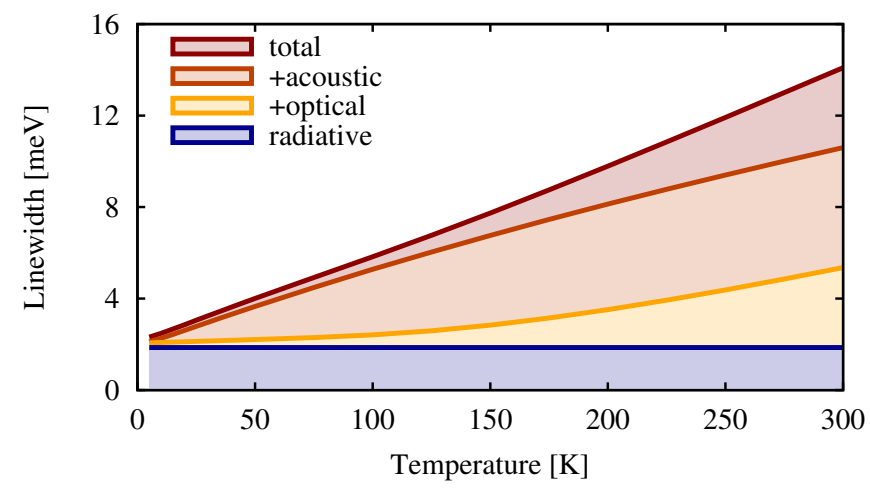

Figure 1. Calculated homogeneous linewidth of the $1 \mathrm{~s}$ A exciton in monolayer $\mathrm{MoS}_{2}$ as a function of temperature. The total linewidth (red) consists of a contributions due to radiative recombination (blue), optical phonon scattering (yellow) and acoustic phonon scattering (orange).

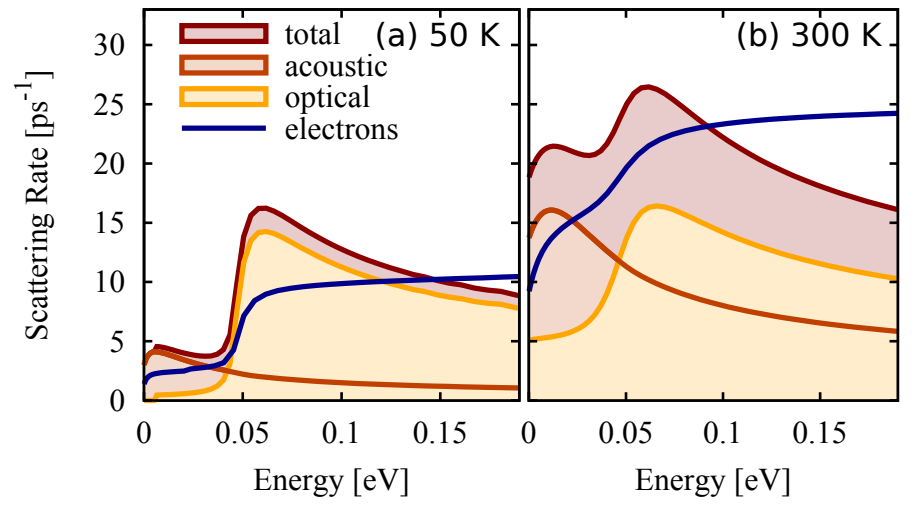

Figure 2. Phonon scattering rates at $50 \mathrm{~K}$ (a) and $300 \mathrm{~K}$ as a function of the exciton kinetic energy in monolayer $\mathrm{MoS}_{2}$. The total scattering rate (red) consists of optical phonon scattering (yellow) and acoustic phonon scattering (orange). As a comparison, the blue line shows the scattering rates of electrons in this material.

In figure 1 we show the homogeneous linewidth (half width at half maximum) of the $1 \mathrm{~s}$ A exciton in monolayer molybdenum disulfide as a function of temperature. The linewidth increases from approximately $2 \mathrm{meV}$ at $0[\mathrm{~K}]$ to $14 \mathrm{meV}$ at room temperature. We find a temperature independent broadening due to radiative recombination of approximately $1.9 \mathrm{meV}$. Acoustic phonons give a linear increasing contribution and optical phonons a super linear increasing. Fitting the data with an effective model

$$
\gamma(T)=\gamma_{0}+\alpha T+\frac{\beta}{\exp \left(\frac{\hbar \Omega}{k T}\right)-1},
$$

we find $\gamma_{0}=1.88 \mathrm{meV}, \alpha=37 \frac{\mathrm{meV}}{\mathrm{K}}$ and $\beta=5 \mathrm{meV}$. The computed linewidth corresponds to a coherence lifetime of $160 \mathrm{fs}$ at $5 \mathrm{~K}$ and $25 \mathrm{fs}$ at room temperature.

In figure 2 we show the scattering rates for the $1 \mathrm{~s} \mathrm{~A} \mathrm{exciton} \mathrm{at} 50 \mathrm{~K}$ (a) and at room temperature (b) in dependence of the exciton kinetic energy $E_{k i n}=\frac{\hbar^{2} \mathbf{Q}^{2}}{2 M}$. Further we show the corresponding electron phonon scattering rates, which are in good agreement with recent theoretical studies. ${ }^{23,24,31}$ We obtain scattering rates in the range of $5-17 \mathrm{ps}^{-1}$ at $50 \mathrm{~K}$ and $16-27 \mathrm{ps}^{-1}$ at $300 \mathrm{~K}$. We find for both temperatures a general decrease with increasing exciton energy except a short increase for very small exciton energies and a more pronounced increase at the optical phonon energy $\hbar \omega^{o p t}=50 \mathrm{meV}$. The first increase can be explained by the enabled emission of acoustic phonons and the enhanced absorption of optical phonons. The large increase at the optical phonon emission energy results of the enabled emission of optical phonons at this energy. The general decrease of the scattering rates can be explained by the exciton phonon coupling 
element equation 12. It depends on the corresponding electron phonon matrix element and on the overlap of initial and final exciton wavefunction. To understand the influence of the pure electron phonon coupling element, we have a look on the electron phonon scattering rates. They exhibit a general increase with the electron kinetic energy and a sharp increase at the optical phonon energy. So the general decrease of the exciton phonon scattering rates at high kinetic energies originates from the overlap integral of initial and final exciton wave function. They differ by $\alpha(\beta) \mathbf{q}^{\prime}$ with the phonon momentum $\mathbf{q}^{\prime}$. Since for higher exciton energies in general larger phonon momenta are required to fulfil the $\delta$-distribution in equation 13 the overlap integral decreases for increasing exciton kinetic energy.

\section{Conclusion}

We have presented a microscopic model revealing the origin of the homogeneous linewidth and coherence lifetime of excitonic resonances in monolayer molybdenum disulfide. We take into account radiative and phonon-induced non-radiative relaxation channels. We find a superlinear increasing homogeneous linewidth from $2 \mathrm{meV}$ at $5 \mathrm{~K}$ to $14 \mathrm{meV}$ at room temperature corresponding to a coherence lifetime of $160 \mathrm{fs}$ and $25 \mathrm{fs}$.

\section{Acknowledgements}

We acknowledge financial support from the Deutsche Forschungsgemeinschaft (DFG) through SFB 951 (G.B. and A.K.) SFB 787 (M.S.), SFB 689 (T.K. and C.S.), GK 1570 (P.N.), Emmy Noether Program (A.C.) and the EU Graphene Flagship (CNECT-ICT-604391)(E.M.,G.B.). This work was further supported by the U.S. Department of Energy, Office of Science, Office of Basic Energy Sciences, with funding at Columbia University through the Energy Frontier Research Center under grant DE-SC0001085 for optical measurements and at SLAC National Accelerator Laboratory through the AMOS program within the Chemical Sciences, Geosciences, and Biosciences Division for data analysis. A.C. gratefully acknowledges funding from the Keck Foundation.

\section{Author contributions}

All authors discussed the results and commented on the manuscript.

\section{Competing financial interests}

The authors declare no competing financial interests.

\section{REFERENCES}

[1] K. F. Mak, C. Lee, J. Hone, J. Shan, and T. F. Heinz, "Atomically thin $\operatorname{mos}_{2}$ : A new direct-gap semiconductor," Phys. Rev. Lett. 105, p. 136805, Sep 2010.

[2] A. Splendiani, L. Sun, Y. Zhang, T. Li, J. Kim, C.-Y. Chim, G. Galli, and F. Wang, "Emerging photoluminescence in monolayer mos2," Nano Letters 10(4), pp. 1271-1275, 2010.

[3] B. Radisavljevic, A. Radenovic, J. Brivio, V. Giacometti, and A. Kis, "Single-layer MoS2 transistors," Nat Nano 6, pp. 147-150, mar 2011. 10.1038/nnano.2010.279.

[4] C. Lee, H. Yan, L. E. Brus, T. F. Heinz, J. Hone, and S. Ryu, "Anomalous lattice vibrations of singleand few-layer mos2," ACS Nano 4(5), pp. 2695-2700, 2010.

[5] K. He, N. Kumar, L. Zhao, Z. Wang, K. F. Mak, H. Zhao, and J. Shan, "Tightly bound excitons in monolayer wse 2 ," Phys. Rev. Lett. 113, p. 026803, Jul 2014.

[6] A. Chernikov, T. C. Berkelbach, H. M. Hill, A. Rigosi, Y. Li, O. B. Aslan, D. R. Reichman, M. S. Hybertsen, and T. F. Heinz, "Exciton binding energy and nonhydrogenic rydberg series in monolayer ws, ," Phys. Rev. Lett. 113, p. 076802, Aug 2014. 
[7] A. Ramasubramaniam, "Large excitonic effects in monolayers of molybdenum and tungsten dichalcogenides," Phys. Rev. B 86, p. 115409, Sep 2012.

[8] A. Singh, G. Moody, K. Tran, M. E. Scott, V. Overbeck, G. Berghäuser, J. Schaibley, E. J. Seifert, D. Pleskot, N. M. Gabor, J. Yan, D. G. Mandrus, M. Richter, E. Malic, X. Xu, and X. Li, "Trion formation dynamics in monolayer transition metal dichalcogenides," Phys. Rev. B 93, p. 041401, Jan 2016.

[9] G. Berghäuser and E. Malic, "Analytical approach to excitonic properties of $\operatorname{mos}_{2}$," Phys. Rev. B 89, p. 125309, Mar 2014.

[10] G. Berghuser, A. Knorr, and E. Malic, "Optical fingerprint of dark 2p-states in transition metal dichalcogenides," 2D Materials 4(1), p. 015029, 2017.

[11] Galan Moody, Chandriker Kavir Dass, Kai Hao, Chang-Hsiao Chen, Lain-Jong Li, Akshay Singh, Kha Tran, Genevieve Clark, Xiaodong Xu, Gunnar Berghauser, Ermin Malic, Andreas Knorr, and Xiaoqin $\mathrm{Li}$, "Intrinsic homogeneous linewidth and broadening mechanisms of excitons in monolayer transition metal dichalcogenides," Nat Commun 6, sep 2015.

[12] S. Koirala, S. Mouri, Y. Miyauchi, and K. Matsuda, "Homogeneous linewidth broadening and exciton dephasing mechanism in MoTe 2 ," Phys. Rev. B 93, p. 075411, Feb 2016.

[13] M. Selig, G. Berghäuser, A. Raja, P. Nagler, C. Schüller, T. F. Heinz, T. Korn, A. Chernikov, E. Malic, and A. Knorr, "Excitonic linewidth and coherence lifetime in monolayer transition metal dichalcogenides," Nature Communications 7, p. 13279, nov 2016.

[14] H. Wang, C. Zhang, W. Chan, C. Manolatou, S. Tiwari, and F. Rana, "Radiative lifetimes of excitons and trions in monolayers of the metal dichalcogenide $\operatorname{mos}_{2}$," Phys. Rev. B 93, p. 045407, Jan 2016.

[15] A. O. Slobodeniuk and D. M. Basko, "Exciton-phonon relaxation bottleneck and radiative decay of thermal exciton reservoir in two-dimensional materials," Phys. Rev. B 94, p. 205423, Nov 2016.

[16] E. Malic and A. Knorr, Graphene and Carbon Nanotubes: Ultrafast Optics and Relaxation Dynamics, 1st ed. (Wiley-VCH, Berlin, 2013).

[17] H. Haug and S. W. Koch, Quantum Theory of the Optical and Electronic Properties of Semiconductors, 5th ed. (World Scientific Publishing Co. Pre. Ltd., Singapore, 2004).

[18] V. M. Axt and T. Kuhn, "Femtosecond spectroscopy in semiconductors: a key to coherences, correlations and quantum kinetics," Reports on Progress in Physics 67(4), p. 433, 2004.

[19] M. Kira and S. Koch, "Many-body correlations and excitonic effects in semiconductor spectroscopy," Progress in Quantum Electronics 30(5), pp. 155 - 296, 2006.

[20] E. Malic, T. Winzer, E. Bobkin, and A. Knorr, "Microscopic theory of absorption and ultrafast manyparticle kinetics in graphene," Phys. Rev. B 84, p. 205406, Nov 2011.

[21] A. Thränhardt, S. Kuckenburg, A. Knorr, T. Meier, and S. W. Koch, "Quantum theory of phononassisted exciton formation and luminescence in semiconductor quantum wells," Phys. Rev. B 62, pp. 2706-2720, Jul 2000.

[22] A. Kormnyos, G. Burkard, M. Gmitra, J. Fabian, V. Zlyomi, N. D. Drummond, and V. Falko, "k p theory for two-dimensional transition metal dichalcogenide semiconductors," 2D Materials 2(2), p. 022001, 2015.

[23] X. Li, J. T. Mullen, Z. Jin, K. M. Borysenko, M. Buongiorno Nardelli, and K. W. Kim, "Intrinsic electrical transport properties of monolayer silicene and $\operatorname{mos}_{2}$ from first principles," Phys. Rev. B 87, p. 115418, Mar 2013.

[24] Z. Jin, X. Li, J. T. Mullen, and K. W. Kim, "Intrinsic transport properties of electrons and holes in monolayer transition-metal dichalcogenides," Phys. Rev. B 90, p. 045422, Jul 2014.

[25] Y. Li, A. Chernikov, X. Zhang, A. Rigosi, H. M. Hill, A. M. van der Zande, D. A. Chenet, E.-M. Shih, J. Hone, and T. F. Heinz, "Measurement of the optical dielectric function of monolayer transitionmetal dichalcogenides: $\operatorname{mos}_{2}, \mathrm{MoSe}_{2}, \mathrm{ws}_{2}$, and WSe 2 ," Phys. Rev. B 90, p. 205422, Nov 2014.

[26] A. Steinhoff, J.-H. Kim, F. Jahnke, M. Rösner, D.-S. Kim, C. Lee, G. H. Han, M. S. Jeong, T. O. Wehling, and C. Gies, "Efficient Excitonic Photoluminescence in Direct and Indirect Band Gap Monolayer MoS2," Nano Letters 15(10), pp. 6841-6847, 2015. 
[27] M. Feierabend, G. Berghäuser, A. Knorr, and E. Malic, "Out of the dark and into the light: towards dark-state based chemical sensors," arXiv:1612.03006, 2016.

[28] X.-X. Zhang, Y. You, S. Y. F. Zhao, and T. F. Heinz, "Experimental evidence for dark excitons in monolayer wse 2 ," Phys. Rev. Lett. 115, p. 257403, Dec 2015.

[29] R. Schmidt, G. Berghuser, R. Schneider, M. Selig, P. Tonndorf, E. Mali, A. Knorr, S. Michaelis de Vasconcellos, and R. Bratschitsch, "Ultrafast coulomb-induced intervalley coupling in atomically thin ws2," Nano Letters 16(5), pp. 2945-2950, 2016.

[30] G. Plechinger, P. Nagler, A. Arora, R. Schmidt, A. Chernikov, A. G. del Águila, P. C. Christianen, R. Bratschitsch, C. Schüller, and T. Korn, "Trion fine structure and coupled spin-valley dynamics in monolayer tungsten disulfide," Nature Communications 7, p. 12715, sep 2016.

[31] K. Kaasbjerg, K. S. Thygesen, and K. W. Jacobsen, "Phonon-limited mobility in $n$-type single-layer $\operatorname{mos}_{2}$ from first principles," Phys. Rev. B 85, p. 115317, Mar 2012. 